\title{
Monoclonal Antibody F19
}

National Cancer Institute

\section{Source}

National Cancer Institute. Monoclonal Antibody F19. NCI Thesaurus. Code C2514.

A murine monoclonal antibody (MoAb) against human fibroblast activation protein (FAP).

FAP is a $95 \mathrm{kDa}$ cell surface glycoprotein and an inducible tumor stromal antigen of epithelial cancers and of a subset of soft tissue sarcomas. FAP shows a very limited distribution pattern in normal tissues, thereby MoAb F19 has possible diagnostic and therapeutic applications in epithelial cancers. 